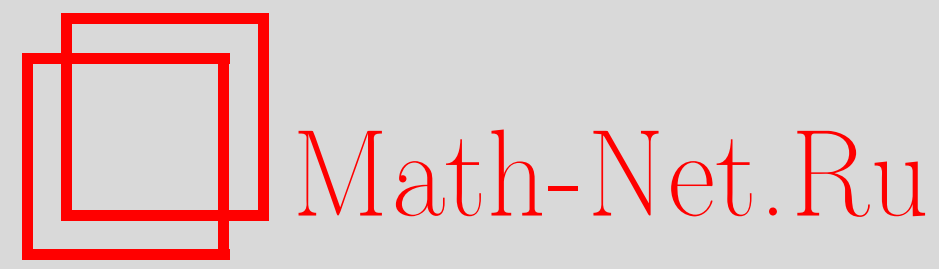

А. Н. Чупрунов, О сходимости случайных ломаных с нормировками типа Стьюдента, Теория вероятн. и ее примен., 1996, том 41, выпуск 4, 914-919

DOI: https://doi.org/10.4213/tvp3282

Использование Общероссийского математического портала Math-Net.Ru подразумевает, что вы прочитали и согласны с пользовательским соглашением http://www. mathnet.ru/rus/agreement

Параметры загрузки:

IP : 35.173 .219 .12

26 апреля 2023 г., 02:11:26

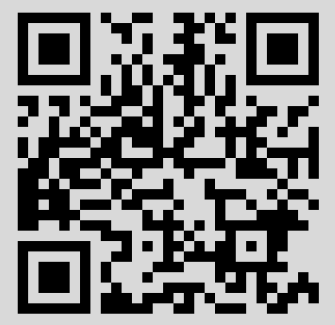


$1996 \mathrm{~T}$

ЧУПРУНОВ А. Н.*

\section{О СХОДИМОСТИ СЛУЧАЙНЫХ ЛОМАНЫХ С НОРМИРОВКАМИ ТИПА СТЬЮДЕНТА ${ }^{1)}$}

Приведены условия, обеспечивающие сходимость случайных ломаных и случайных ступенчатых функций, определенных суммами независимых случайных величин с нормировками типа Стьюдента к винеровскому процессу и $p$-устойчивому случайному процессу с независимыми прирашениями. Особое внимание уделено случаю, когда рассматриваемые случайные величины являются наблюдениями случайного процесса в случайные моменты времени.

Клюиевые слова и Фразы: суммы независимых случайных величин, сходимость по распределению, винеровский процесс, однородный случайный процесс с независимыми приращениями.

Пусть $(\Omega, \mathfrak{A}, \mathbf{P})$ - вероятностное пространство, $(T, \mathfrak{B})-$ измеримое пространство, $X_{n}(t), t \in T, n \in \mathbf{N},-$ случайные процессы, определенные на $(\Omega, \mathfrak{A}, \mathbf{P})$ и измеримые как функции двух аргументов относительно произведения $\sigma$-алгебр $\mathfrak{A} \times \mathfrak{B}$. Обозначим через $X_{n i}, i, n \in \mathbf{N}$, независимые копии $X_{n}$. Пусть $\xi$ и $\xi_{n}, n \in \mathbf{N},-$ независимые одинаково распределенные случайные величины со значениями в $(T, \mathfrak{B})$, определенные на другом вероятностном пространстве $\left(\Omega_{1}, \mathfrak{A}_{1}, \mathbf{P}_{1}\right)$.

В [1] получены условия сходимости по распределению сумм и максимумов от случайных величин $X_{n i}\left(\xi_{i}\left(\omega_{1}\right)\right)$ для почти всех $\omega_{1} \in \Omega_{1}$. В [2] изучаются обобщения результатов [1] на случайные процессы $X_{n i}$, принимающие значения в банаховом пространстве. В [3] изучается сходимость эмпирических случайных процессов, определенных случайными величинами $X_{n i}\left(\xi_{i}\left(\omega_{1}\right)\right)$. Здесь мы продолжим эти исследования.

Случайные величины $X_{n i}\left(\xi_{i}\left(\omega_{1}\right)\right)$ возникают в некоторых задачах, связанных с применениями теории вероятностей. Рассмотрим, например, выборку из $m$ работников цеха. С $k$-м работником из этой выборки связывается пара чисел $\left(x_{k}, t_{k}\right)$, где $x_{k}$ - числовая характеристика рассматриваемого параметра здоровья, а $t_{k}$ - число лет, проработанных работником в этом цехе. Пусть $X(t), t \in \mathbf{R}^{+},-$случайный процесс, описывающий эволюцию этого параметра здоровья у работников щеха $(X(t)-$ случайная величина, характеризуюшцая параметр здоровья у людей, проработавших ровно $t$ лет в этом цехе). Тогда числа $x_{k}$ можно рассматривать как наблюдения случайного процесса $X(t)$ в случайные моменты времени $t_{k}, 1 \leqslant k \leqslant m$. Итак, наша задача свелась к изучению случайного процесса $X(t)$ по наблюдениям $\left(x_{k}, t_{k}\right)$, $1 \leqslant k \leqslant m$. При этом мы считаем, что $t_{k}$ - независимые наблюдения одной и той же случайной величины $\xi$.

Пусть $D$ - подмножество вещественной прямой $\mathbf{R}, L$ - некоторое линейное пространство функций на $D$, наделенное метризуемой топологией, $H(y), H_{n}(y), y \in D$, случайные процессы, почти все траектории которых принадлежат $L$. Говорят, что процессы $H_{n}(y)$ сходятся по распределению к процессу $H(y)$ в $L$ (и обозначают $\left.H_{n} \stackrel{\mathrm{d}}{\longrightarrow} H, n \rightarrow \infty\right)$, если распределения случайных процессов $H_{n}$ сходятся слабо в $L$ к распределению случайного процесса $H$. Здесь в качестве пространства $L$ мы будем рассматривать пространство непрерывных функций $C[0,1]$ или пространство

*НИИ математики и механики, ул. Университетская, 17, 420008 Казань, Татарcraн.

1) Работа выполнена при поддержке Российского фонда фундаментальных исследований (грант № 96-01-01265). 
Скорохода $\mathcal{D}[0,1]$. Символом $\stackrel{d}{\longrightarrow}$ будем обозначать также сходимость по распределению случайных элементов. Символом $\stackrel{d}{=}$ будем обозначать равенство распределений случайных величин.

Пусть случайный процесс $H(y), y \in[0,1]$, определен в точках $y=x_{k}, 1 \leqslant k \leqslant n$ $0=x_{1}<x_{2}<\cdots<x_{n}=1$. Будем говорить, что в остальных точках отрезка $[0,1]$ случайный процесс $H(y)$ определен по линейности, если для любой точки $x \in[0,1]$, $x_{i}<x<x_{i+1}, Z(x)=\alpha Z\left(x_{i}\right)+(1-\alpha) Z\left(x_{i+1}\right)$, где $\alpha$ - такое положительное число, что $x=\alpha x_{i}+(1-\alpha) x_{i+1}$.

Пусть $Z, Z_{n}, n \hat{\epsilon} \mathbf{N}$ - случайные элементы со значениями в банаховом пространстве $B, \eta, \eta_{n}, n \in \mathbf{N}$, - случайные величины. Уже в одномерном случае легко построить такие $\eta, \eta_{n}, n \in \mathbb{N}$, и $Z, Z_{n}, n \in \mathbf{N}$, что $\eta_{n} \stackrel{\mathrm{d}}{\longrightarrow} \eta, n \rightarrow \infty$, и $Z_{n} \stackrel{\mathrm{d}}{\longrightarrow} Z$, $n \rightarrow \infty$, но последовательность $\eta_{n} Z_{n}$ не сходится по распределению. Тем не менее справедливо следующее утверждение.

Лемма 1. Пусть $\eta_{n} \stackrel{\mathrm{d}}{\longrightarrow} C, n \rightarrow \infty$, где $C-$ некоторое число, $u Z_{n} \stackrel{\mathrm{d}}{\longrightarrow} Z$, $n \rightarrow \infty$. Тогда $\eta_{n} Z_{n} \stackrel{\mathrm{d}}{\longrightarrow} C Z, n \rightarrow \infty$.

Применим лемму 1 к случайным элементам, рассматриваемым в теореме Донскера-Прохорова.

Пусть $\xi_{n k}, 1 \leqslant k \leqslant k_{n}, n \in \mathbf{N}$, - независимые в каждой серии бесконечно малые случайные величины. Напомним условие Линдеберга: случайные величины $\xi_{n k}-$ центрированные, $\sigma_{n i}^{2}=\mathbf{E}\left(\xi_{n i}\right)^{2}<\infty$ для всех $1 \leqslant i \leqslant k_{n}, n \in \mathbf{N}$, $\max _{1 \leqslant i \leqslant k_{n}} \sigma_{n i}^{2} / b_{n} \longrightarrow 0, n \rightarrow \infty$, где $b_{n}=\sum_{i=1}^{k_{n}} \sigma_{n i}^{2}, n \in \mathbf{N}$, и для каждого $\varepsilon>0$

$$
\left(b_{n}\right)^{-1} \sum_{i=1}^{k_{n}} \mathbf{E}\left(\xi_{n i}\right)^{2} I\left\{\left|\xi_{n i}\right| \geqslant \varepsilon\left(b_{n}\right)^{1 / 2}\right\} \rightarrow 0, \quad n \rightarrow \infty .
$$

Определим случайные процессы $U_{n}(x), x \in[0,1] . \quad$ При $x=\left(b_{n}\right)^{-1} \sum_{i=1}^{k} \sigma_{n i}^{2}$, $1 \leqslant k \leqslant k_{n}$, положим $U_{n}(x)=\sum_{i=1}^{k} \xi_{n i}$. В остальных точках отрезка $[0,1]$ случайные процессы $U_{n}(x)$ определены по линейности. Везде далее мы будем предполагать, что $k_{n} \rightarrow \infty, n \rightarrow \infty$.

Теорема Прохорова [4]. Пусть выполнено условие Линдеберга. Тогда $\left(b_{n}\right)^{-1 / 2} U_{n} \stackrel{\mathrm{d}}{\longrightarrow} W, n \rightarrow \infty$, в $C[0,1]$, әде $W-$ винеровский процесс.

Обозначим через $C_{r n k}$ и $B_{r n k}$ тяжелые $r$-центры и $r$-разбросы случайных величин $\xi_{n k}$ (см. определения $r$-центров и $r$-разбросов в монографии [5]), пусть $B_{n}=$ $\sum_{i=1}^{k_{n}} B_{r n i}$.

Определим случайные процессы $V_{n}(x), x \in[0,1]$. IІри $x=\left(B_{n}\right)^{-1} \sum_{i=1}^{k} B_{r n i}$, $1 \leqslant k \leqslant k_{n}$, положим $V_{n}(x)=\sum_{i=1}^{k} \xi_{n i}$. В остальных точках отрезка $[0,1]$ случайные процессы $V_{n}(x)$ определены по линейности.

Теорема Круглова [6]. Пусть $C_{r n k}=0,1 \leqslant k \leqslant k_{n}, u B_{n}=1, n \in \mathbf{N}$. Тогдa $V_{n} \stackrel{\mathrm{d}}{\rightarrow} W, n \rightarrow \infty$, в $C[0,1]$.

Из теоремы Прохорова и теоремы Круглова, используя лемму 1 , получаем следующий результат.

Предложение 1. Пусть для всех $n \in \mathbf{N}$ выполнено равенство

$$
\mathbf{P}\left\{\max _{1 \leqslant i \leqslant k_{n}}\left|\xi_{n i}\right|=0\right\}=0
$$

(1) Пусть выполнено условие Линдеберга. Тогда

$$
\left(\sum_{i=1}^{k_{n}}\left(\xi_{n i}\right)^{2}\right)^{-1 / 2} U_{n} \stackrel{d}{\longrightarrow} W, \quad n \rightarrow \infty, \quad 6 \quad C[0,1] .
$$


(2) $\Pi_{y c m b} C_{r n k}=0,1 \leqslant k \leqslant k_{n}, n \in \mathbf{N}, B_{n} \rightarrow B, n \rightarrow \infty, u \infty>B>0$. Toгda

$$
\left(\sum_{i=1}^{k_{n}}\left(\xi_{n i}\right)^{2}\right)^{-1 / 2} V_{n} \stackrel{\mathrm{d}}{\longrightarrow} W, \quad n \rightarrow \infty, \quad \text { в } C[0,1] .
$$

Приведем аналоги предложения 1 для случайных величин $X_{n i}\left(\xi_{i}\left(\omega_{1}\right)\right)$. Пусть

$$
S_{n x}^{*}\left(\omega_{1}\right)=\sum_{i=1}^{\left[k_{n} x\right]} X_{n i}\left(\xi_{i}\left(\omega_{1}\right)\right), \quad 0 \leqslant x \leqslant 1, \quad \omega_{1} \in \Omega_{1}, \quad n \in \mathbf{N} .
$$

Рассмотрим случайные процессы

$$
S_{n}^{\prime}\left(x, \omega_{1}\right)=S_{n x}^{*}\left(\omega_{1}\right)+\left\{k_{n} x\right\} X_{n\left(\left[k_{n} x\right]+1\right)}\left(\xi_{\left[k_{n} x\right]+1}\left(\omega_{1}\right)\right), \quad 0 \leqslant x \leqslant 1,
$$

параметризованные элементами $\boldsymbol{\omega}_{1} \in \Omega_{1}$. Все траектории случайных процессов $S_{n}^{\prime}\left(x, \omega_{1}\right)$ лежат в пространстве $C[0,1]$.

Пусть $\tau>0$. Положим

$$
A(t)=\sup _{n \in \mathbb{N}} k_{n}\left|\mathbf{E} X_{n}{ }^{\prime}(t) I\left\{\left|X_{n}(t)\right|<\tau\right\}\right|, \quad B(t)=\sup _{n \in \mathbf{N}} k_{n} \mathbf{E} \frac{\left(X_{n}(t)\right)^{2}}{1+\left(X_{n}(t)\right)^{2}}, \quad t \in T,
$$

символом $\mathbf{E}_{1}$ обозначим математическое ожидание относительно вероятности $\mathbf{P}_{1}$, пусть $\gamma\left(\sigma^{2}\right)$ - симметричная гауссовская случайная величина с дисперсией $\sigma^{2}$.

Лемма 2. Пусть $S_{n}(t) \stackrel{\mathrm{d}}{\longrightarrow} \gamma\left(\sigma^{2}(t)\right), n \rightarrow \infty$, для каждого $t \in T, \mathbf{E}_{1} \sigma^{2}(\dot{\xi})=b$, причем $\infty>b>0, \mathbf{E}_{1} A(\xi)<\infty u \mathbf{E}_{1} B(\xi)<\infty$. Тогда $S_{n}^{\prime}\left(x, \omega_{1}\right) \stackrel{\mathrm{d}}{\longrightarrow} b^{1 / 2} W, n \rightarrow \infty$, в $C[0,1]$ dag noumu всеx $\omega_{1} \in \Omega_{1}$.

Пусть $Y(t), Y_{n}(t), t \in T, n \in \mathbf{N},-$ независимые одинаково распределенные случайные процессы, определенные на $(\Omega, \mathfrak{A}, \mathbf{P})$ и как функции двух аргументов измеримые относительно произведения $\sigma$-алгебр $\mathfrak{A} \times \mathfrak{B}$. Рассмотрим случайные процессы

$$
S_{n}^{\prime \prime}\left(x, \omega_{1}\right)=n^{-1 / 2}\left(\sum_{i=1}^{[n x]} Y_{i}\left(\xi_{i}\left(\omega_{1}\right)\right)+\{n x\} Y_{\left[k_{n} x\right]+1}\left(\xi_{\left[k_{n} x\right]+1}\left(\omega_{1}\right)\right)\right), \quad 0 \leqslant x \leqslant 1,
$$

параметризованные элементами $\omega_{1} \in \Omega_{1}$.

Следствие 1. Пусть $\mathbf{E} Y(t)=0$ для всех $t \in T$ u $0<b<\infty$, где $b=\mathbf{E}_{1} \mathbf{E}(Y(\xi))^{2}$. Toгдa $S_{n}^{\prime \prime}\left(x, \omega_{1}\right) \stackrel{\mathrm{d}}{\longrightarrow} b^{1 / 2} W, n \rightarrow \infty$, в $C[0,1]$ для noumu всех $\omega_{1} \in \Omega_{1}$.

Из леммы 2 и следствия 1 , ввиду леммы 1 , вытекают, соответственно, теорема 1 и следствие 2.

Теорема 1. Пусть выполнены условия леммы 2 и для всех $n \in \mathbf{N}$ справедливо равеяство

$$
\mathbf{P}_{1}\left\{\omega_{1} \in \Omega_{1}: \mathbf{P}\left\{X_{n}(\xi)=0\right\}>0\right\}=0
$$

Tozda

$$
\left(\sum_{i=1}^{k_{n}} X_{n i}\left(\xi_{i}\left(\omega_{1}\right)\right)\right)^{-1 / 2} S_{n}^{\prime}\left(x, \omega_{1}\right) \stackrel{\mathrm{d}}{\longrightarrow} W, \quad n \rightarrow \infty
$$

в $C[0,1]$ для почmu всех $\omega_{1} \in \Omega_{1}$.

Следствие 2. Пусть выполнены условия следствия $1, b=\mathbf{E}_{1} \mathbf{E}(Y(\xi))^{2} u$ $\mathbf{P}_{1}\left\{\omega_{1} \in \Omega_{1}: \mathbf{P}\{Y(\xi)=0\}>0\right\}=0$. Tогдa

$$
\left(\sum_{i=1}^{n} Y_{i}\left(\xi_{i}\left(\omega_{1}\right)\right)\right)^{-1 / 2} S_{n}^{\prime \prime}\left(x, \omega_{1}\right) \stackrel{\mathrm{d}}{\longrightarrow} W, \quad n \rightarrow \infty,
$$


в $C[0,1]$ для почmu всех $\omega_{1} \in \Omega_{1}$.

Рассмотрим случайные процессы $S_{n}^{t}\left(x, \omega_{1}\right), 0 \leqslant x \leqslant 1, n \in \mathbf{N}$, параметризованные $\omega_{1} \in \Omega_{1}$. При $x=m / k_{n}, 0 \leqslant m \leqslant k_{n}$, полагаем

$$
S_{n}^{t}\left(x, \omega_{1}\right)=2 \sum_{j<i \leqslant m} X_{n j}\left(\xi_{j}\left(\omega_{1}\right)\right) X_{n i}\left(\xi_{i}\left(\omega_{1}\right)\right) .
$$

В остальных точках $x \in[0,1]$ случайные процессы $S_{n}^{t}\left(x, \omega_{1}\right)$ определены по линейности:

$$
S_{n}^{t}\left(x, \omega_{1}\right)=S_{n}^{t}\left(\frac{\left[x k_{n}\right]}{k_{n}}, \omega_{1}\right)+\left\{x k_{n}\right\}\left(S_{n}^{t}\left(\frac{\left[x k_{n}\right]+1}{k_{n}}, \omega_{1}\right)-S_{n}^{t}\left(\frac{\left[x k_{n}\right]}{k_{n}}, \omega_{1}\right)\right) .
$$

Обозначим через $I$ такой элемент пространства $C[0,1]$, что $I(x)=x, x \in[0,1]$.

Лемма 3. Пусть выполнены условия леммы 2. Тогда $S_{n}^{t}\left(x, \omega_{1}\right) \stackrel{\mathrm{d}}{\longrightarrow} b\left(W^{2}-I\right)$, $n \rightarrow \infty$, в $C[0,1]$ для почти всех $\omega_{1} \in \Omega_{1}$.

Рассмотрим следующие случайные процессы, параметризованные элементами $\omega_{1} \in \Omega_{1}$ :

$$
\begin{aligned}
S_{n}^{\&}\left(x, \omega_{1}\right)=2( & \sum_{j<i \leqslant[n x]} Y_{i}\left(\xi_{i}\left(\omega_{1}\right)\right) Y_{j}\left(\xi_{j}\left(\omega_{1}\right)\right) \\
& \left.+\{n x\} \sum_{\substack{[n x]<i \leqslant j n]+1, j<i \leqslant\{n x]+1}} Y_{i}\left(\xi_{i}\left(\omega_{1}\right)\right) Y_{j}\left(\xi_{j}\left(\omega_{1}\right)\right)\right), \quad 0 \leqslant x \leqslant 1 .
\end{aligned}
$$

При $X_{n i}=n^{-1 / 2} Y_{i}$ из леммы 3 выводим

Следствие 3. Пусть выполнень условия следствия 1 . Тогда $n^{-1} S_{n}^{\&}\left(x, \omega_{1}\right) \stackrel{d}{\longrightarrow}$ $b\left(W^{2}-I\right), n \rightarrow \infty$, в $C[0,1]$ для почти всех $\omega_{1} \in \Omega_{1}$.

Из леммы 3 и следствия 3 , ввиду леммы 1 , соответственно, вытекают теорема 2 и следствие 4.

Теорема 2. Пусть выполнены условия теоремы 1. Тогда

$$
\left(\sum_{i=1}^{k_{n}}\left(X_{n i}\left(\xi_{i}\left(\omega_{1}\right)\right)\right)^{2}\right)^{-1} S_{n}^{t}\left(x, \omega_{1}\right) \stackrel{\mathrm{d}}{\longrightarrow} W^{2}-I, \quad n \rightarrow \infty,
$$

в $C[0,1]$ длs почmu всех $\omega_{1} \in \Omega_{1}$.

Следствие 4. Пусть выполнены условин следствия 2. Тогда

$$
\left(\sum_{i=1}^{n}\left(Y_{i}\left(\xi_{i}\left(\omega_{1}\right)\right)\right)^{2}\right)^{-1} S_{n}^{\&}\left(x, \omega_{1}\right) \stackrel{\mathrm{d}}{\longrightarrow} W^{2}-I, \quad n \rightarrow \infty
$$

в $C[0,1]$ для почmu всех $\omega_{1} \in \Omega_{1}$.

Пусть $0<p \leqslant 2, \eta, \eta_{n}, n \in \mathbf{N}$, - такие независимые одинаково распределенные случайные величины, центрированные при $p>1$ и симметричные при $p \leqslant 1$, что семейства $\left\{\eta, \eta_{i}\right\},\left\{Y, Y_{i}\right\}$ независимы, $S_{n}^{p}=n^{-1 / p} \sum_{i=1}^{n} \eta_{i}, n \in \mathbf{N}, \gamma_{p}-$ стандартная $p$-устойчивая случайная величина при $0<p<2$ и гауссовская стандартная величина при $p=2$ (т.е. случайная величина $\gamma_{p}$ имеет характеристическую функцию $\widehat{\mathcal{L}}\left(\gamma_{p}\right)(x)=\exp \left(-|x|^{p}\right), x \in \mathbf{R}$, при $0<p<2$ и $\left.\gamma_{2} \stackrel{d}{=} \gamma(1)\right)$. Обозначим через $W_{p}=W_{p}(t), t \in[0,1]$, такой однородный случайный. процесс с независимыми приращениями, что $\mathcal{L}\left(W_{p}(1)\right) \stackrel{\mathrm{d}}{=} \mathcal{L}\left(\gamma_{p}\right)$. Замметим, что при $p=2$ случайный прощесс $W_{p}$ - винеровский процесс $W$. Рассмотрим случайные процессы $S_{n}^{s p}\left(x, \omega_{1}\right)=\sum_{i=1}^{[n x]} \eta_{i} Y_{i}\left(\xi_{i}\left(\omega_{1}\right)\right), 0 \leqslant x \leqslant 1$, параметризованные элементами $\omega_{1} \in \Omega_{1}$. 
Лемма 4. Пусть $0<p<2, S_{n}^{p} \stackrel{\mathrm{d}}{\longrightarrow} \gamma_{p}, n \rightarrow \infty, 0<b<\infty$, аде $b=\operatorname{EE}_{1}|Y(\xi)|^{p}$. Tогда $S_{n}^{s p}\left(x, \omega_{1}\right) \stackrel{\mathrm{d}}{\longrightarrow} b^{1 / p} W_{p}, n \rightarrow \infty$, в $\mathcal{D}[0,1]$ для nочmu всех $\omega_{1} \in \Omega_{1}$.

Из леммы 4 и леммы 1 вытекает следуюший результат.

Теорема 3. Пусть выполяены условия леммы 3 и

$$
\mathbf{P}_{1}\left\{\omega_{1} \in \Omega_{1}: \mathbf{P}\{Y(\xi)=0\}>0\right\}=0 .
$$

Tozda

$$
\left(\sum_{i=1}^{n}\left|Y_{i}\left(\xi_{i}\left(\omega_{1}\right)\right)\right|^{p}\right)^{-1 / p} S_{n}^{s p}\left(x, \omega_{1}\right) \stackrel{\mathrm{d}}{\longrightarrow} W_{p}, \quad n \rightarrow \infty
$$

в $\mathcal{D}[0,1]$ для почти всех $\omega_{1} \in \Omega_{1}$.

Пусть $\xi_{n i}, 1 \leqslant i \leqslant k_{n}, n \in \mathbf{N}$, - такие независимые бесконечно малые случайные величины, что $\left\{\eta_{n}\right\}$ и $\left\{\xi_{n i}\right\}-$ независимые семейства. Пусть $\tau>0$. Положим

$$
\left(\xi_{n i}\right)^{\tau}=\xi_{n i} I\left\{\left|\xi_{n i}\right|<\tau\right\}, \quad \xi_{n 0}=0, \quad a_{n m}=\sum_{i=0}^{m} \mathbf{E}\left|\left(\xi_{n i}\right)^{\tau}\right|^{p}, \quad m \leqslant k_{n}, \quad n \in \mathbf{N} .
$$

Определим случайные процессы $W_{p n}(t), t \in[0,1]$. При $a_{n m} \leqslant t<a_{n(m+1)}, m<k_{n}$, положим $W_{p n}(t)=\sum_{i=1}^{m} \xi_{n i} \eta_{i}$, при $a_{n k_{n}} \leqslant t$ положим $W_{p n}(t)=\sum_{i=1}^{k_{n}} \xi_{n i} \eta_{i}$.

Лемма 5. Пусть $S_{n}^{p} \stackrel{\mathrm{d}}{\longrightarrow} \gamma_{p}, n \rightarrow \infty$. Следуючие условия эквивалентны:

1) $W_{p n} \stackrel{\mathrm{d}}{\longrightarrow} b^{1 / p} W_{p}, n \rightarrow \infty$, в $\mathcal{D}[0,1]$;

2) $\sum_{i=1}^{k_{n}}\left|\xi_{n i}\right|^{p} \longrightarrow b, n \rightarrow \infty$, по вероятrости.

Из леммы 5 и леммы 1 вытекает

Теорема 4. Пусть $S_{n}^{p} \stackrel{\mathrm{d}}{\longrightarrow} \gamma_{p}, n \rightarrow \infty, u W_{p n} \stackrel{\mathrm{d}}{\longrightarrow} b^{1 / p} W_{p}, n \rightarrow \infty$, в $\mathcal{D}[0,1]$, г $\partial e$ $0<b<\infty, u$ для всех $n \in \mathbf{N}$ выполнено равенство

$$
\mathbf{P}\left\{\max _{1 \leqslant i \leqslant k_{n}}\left|\xi_{n i}\right|=0\right\}=0 .
$$

Tогдa

$$
\left(\sum_{i=1}^{k_{n}}\left|\xi_{n i}\right|^{p}\right)^{-1 / p} W_{p n} \stackrel{\mathrm{d}}{\longrightarrow} W_{p}, \quad n \rightarrow \infty, \quad \text { в } \quad \mathcal{D}[0,1]
$$

Пусть

$$
W_{n}^{\prime}\left(a_{n m}\right)=W_{2 n}\left(a_{n m}\right), \quad 1 \leqslant m \leqslant k_{n}, \quad n \in \mathbf{N} .
$$

В остальных точках отрезка $[0,1]$ случайные процессы $W_{n}^{\prime}(x)$ определены по линейности.

Лемма 6. Пусть

$$
\mathrm{E} \eta=0, \quad \mathbf{E} \eta^{2}=1
$$

Следугшие условия эквивалентны:

1) $W_{n}^{\prime} \stackrel{\mathrm{d}}{\longrightarrow} b^{1 / 2} W, n \rightarrow \infty$, в $C[0,1]$;

2) $\sum_{i=1}^{k_{n}}\left|\xi_{n i}\right|^{2} \longrightarrow b, n \rightarrow \infty$, по вероятности.

Теорема 5. Пусть

$$
\mathbf{E} \eta=0, \quad \mathbf{E} \eta^{2}=1 \quad u \quad W_{n}^{\prime} \stackrel{\mathrm{d}}{\longrightarrow} b^{1 / 2} W, \quad n \rightarrow \infty,
$$

в $C[0,1]$, аде $0<b<\infty$, и длн всех $n \in \mathbf{N}$ ияеем

$$
\mathbf{P}\left\{\max _{1 \leqslant i \leqslant k_{n}}\left|\xi_{n i}\right|=0\right\}=0 .
$$


Tozda

$$
\left(\sum_{i=1}^{k_{n}}\left|\xi_{n i}\right|^{2}\right)^{-1 / 2} W_{n}^{\prime} \stackrel{\mathrm{d}}{\longrightarrow} W, \quad n \rightarrow \infty, \quad \text { в } C[0,1] .
$$

3 а м е ч а н е 1. В идейном плане доказательства лемм 2,3 и 6 близки к доказательству теоремы Прохорова. А. В. Скороход (см., например, [7, с. 547]) получил условия сходимости случайных ступенчатых функций, определенных суммами независимых случайных величин, к однородному случайному процессу с независимыми приращениями. В идейном плане доказательства лемм 4 и 5 близки к доказательству теоремы Скорохода.

3 а м е ч а н и е 2. Заметим, что условия: «для всех $n \in \mathbf{N}$ выполнено равенство $\mathbf{P}\left\{\max _{1 \leqslant i \leqslant k_{n}}\left|\xi_{n i}\right|=0\right\}=0 \gg$ в предложении 1 , теоремах 4 и 5 ; «для всех $n \in \mathbf{N}$ выполнено равенство $\mathbf{P}_{1}\left\{\omega_{1} \in \Omega_{1}: \mathbf{P}\left\{X_{n}(\xi)=0\right\}>0\right\}=0 \gg$ в теоремах 2 и 3 ; « $\mathbf{P}_{1}\left\{\omega_{1} \in \Omega_{1}: \mathbf{P}\{Y(\xi)=0\}>0\right\}=0 »$ в следствиях 2 и 4 и теореме 3 , обеспечивают почти наверное определенность рассматриваемых случайных элементов и не имеют существенного использования в доказательствах. Поэтому если расширить понятие сходимости по распределению на не обязательно почти всюду определенные случайные величины, то эти условия в перечисленных выше теоремах являются излишними. Приведем один из вариантов такого расширения: последовательность $Z_{n}$ не обязательно почти всюду определенных случайных элементов в банаховом пространстве $B$ сходится по распределению к случайному элементу $Z$ со значением в $B$ тогда и только тогда, когда

а) для каждого $\varepsilon>0$ найдутся такие $n_{\varepsilon} \in \mathbf{N}$ и компакт $K_{\varepsilon} \subset B$, что $\mathbf{P}\left\{Z_{n} \in\right.$ $\left.K_{\varepsilon}\right\}>1-\varepsilon$ для всех $n>n_{\varepsilon}$

б) для каждого $x^{\prime} \in B^{\prime}$, для кажцой такой точки $y \in \mathbf{R}$, что функция распределения случайной величины $x^{\prime}(Z)$ непрерывна в $y$, числовая последовательность $\mathbf{P}\left\{x^{\prime}\left(Z_{n}\right) \leqslant y, x^{\prime}\left(Z_{n}\right)\right.$ определена $\}$ сходится к $\mathbf{P}\left\{x^{\prime}(Z) \leqslant y\right\}$.

Заметим, что в силу критерия слабой сходимости Прохорова для почти всюду определенных случайных элементов это определение совпадает с обычным определением сходимости по распределению.

\section{СПИСОК ЈИТЕРАТУРЫ}

1. Чупрунов A. Н. О сходимости по распределению сумм и максимумов независимых случайных величин со случайными параметрами. - Liet. Matem. Rink., 1995 , v. 35, № 1, p. 52-64.

2. Чупрунов A. H. О сходимости по распределению эмпирических процессов определенных независимыми случайными процессами. - Liet. Matem. Rink., 1995, v. 35, № 2, p. 171-180.

3. Чупрунов $A$. Н. О сходимости по распределению почти всех сумм независимых банаховозначных случайных элементов. - Изв. ВУЗов. Математика, 1994, № 11, p. $83-87$.

4. Прохоров Ю. В. Сходимость случайных процессов и предельные теоремы теории вероятностей. - Теория вероятн. и ее примен., 1956, т. 1, в. 2, с. 117-238.

5. Золотарев B. M. Современная теория суммирования независимых случайных величин. М.: Наука, 1986, 415 с.

6. Круглов $B$. М. Слабая сходимость случайных люманых к винеровскому процессу. - Теория вероятн. и ее примен., 1985, т. 30, в. 2, с. 209-218.

7. Гихман И. И., Скороход А. В. Введение в теорию случайных процессов. М.: Наука, 1977, 568 c. 